\title{
Intensity-modulated radiation therapy combined with concomitant temozolomide for brain metastases from lung adenocarcinoma
}

\author{
JINLI LI $^{1 *}$, XIAOYAN CHAI $^{2 *}$, YING CAO $^{1}$, XIAOCHU HU $^{1}$, \\ HONGYU ZHU ${ }^{1}$, JIANPING WANG ${ }^{1}$ and YIWEI WU ${ }^{3}$ \\ ${ }^{1}$ Department of Radiation Oncology, The Affiliated Hospital of Soochow University, Suzhou, Jiangsu 215006; \\ ${ }^{2}$ Department of Oncology, Suzhou Science and Technology Town Hospital, Suzhou, Jiangsu 215153; \\ ${ }^{3}$ Department of Nuclear Medicine, The Affiliated Hospital of Soochow University, Suzhou, Jiangsu 215006, P.R. China
}

Received August 29, 2017; Accepted June 19, 2018

DOI: $10.3892 / \mathrm{ol} .2018 .9171$

\begin{abstract}
Short-term efficacy, adverse effects and the impact on quality of life (QoL) of a concomitant treatment with intensity-modulated radiation therapy (IMRT) and temozolomide (TMZ) in patients with brain metastases (BMs) from lung adenocarcinoma were evaluated. This study sought to confirm the benefit of adding TMZ to IMRT in patients with BMs from lung adenocarcinoma. Nine patients were enrolled and received a dose of $30 \mathrm{~Gy}$ in 10 daily fractions to clinical tumor volume (CTV) according to IMRT, then additional dose of 9 Gy in 3 fractions of IMRT was delivered to gross tumor volume (GTV) only with concomitant TMZ $\left(75 \mathrm{mg} / \mathrm{m}^{2} /\right.$ day) orally during RT for 3 weeks. One patient achieved complete response (CR) (11.1\%), 6 patients obtained partial response (PR) $(66.7 \%)$, and there were no patients in progression. Therefore, objective response (OR) reached $77.8 \%$. The main adverse effects included neutropenia, anemia, vomiting, fatigue and dizziness. Grade $\geq 3$ of hematologic toxicities did not occur. However, the other 9 patients who received only intensity-modulated radiation had much worse results. The CR was 0 , PR rate was $44.4 \%$, OR rate was $44.4 \%$. The results indicated that the benefit of adding TMZ to IMRT was confirmed in patients with BMs from lung adenocarcinoma. The treatment was active, a significant $\mathrm{OR}$ was
\end{abstract}

Correspondence to: Dr Jianping Wang, Department of Radiation Oncology, The Affiliated Hospital of Soochow University, 899 Pinghai Road, Gusu, Suzhou, Jiangsu 215006, P.R. China E-mail: wangjianpingsz@163.com

Dr Yiwei Wu, Department of Nuclear Medicine, The Affiliated Hospital of Soochow University, 899 Pinghai Road, Gusu, Suzhou, Jiangsu 215006, P.R. China

E-mail: wuyiwei3988@gmail.com

*Contributed equally

Key words: brain metastases, lung adenocarcinoma, non-small cell lung cancer, intensity-modulated radiation therapy, temozolomide observed, and achieved an improvement in QoL demonstrated by QoL grade $(\mathrm{p}<0.05)$.

\section{Introduction}

Brain metastasis (BM) is one of the important causes of death in patients with non-small cell lung cancer (NSCLC). The natural course of the disease is 1-3 months, and the median survival time is only 4 months (1). The incidence of BMs has increased in recent years, and is associated with poor prognosis. Treatment of metastatic brain tumors for NSCLC presents a particular challenge: the majority of patients with BMs present multiple lesions. The standard treatment is whole brain radiotherapy (WBRT), which may help to improve the local control rate and alleviate the clinical symptoms, and the median survival time extended to 3-6 months. The recurrence rate of intracranial tumors following WBRT is $52 \%$, so it is necessary to give a higher therapeutic dose to the BMs, in order to improve the local control and survival rate of the tumor $(2,3)$. Historically, the treatment options of local tumor pushing with BMs include three-dimensional conformal radiotherapy (3D-CRT), intensity-modulated radiation therapy (IMRT) and stereotactic radiosurgery (SRS). In recent years, IMRT has played an important role in the treatment of radiation therapy, which has attracted wide attention. Therefore, IMRT has been proposed for the treatment of BMs. In particular, IMRT was evaluated in several planning irradiation studies, mainly in order to protect normal brain tissue, to simultaneously boost the local dose to brain lesions during WBRT (4-7).

Systemic chemotherapy in the treatment of BMs is limited and it has been widely controverted. The limited ability of most chemotherapeutic agents to cross the blood-brain barrier (BBB) is believed to be one of the principal reasons. The agents that have difficulty in reaching the central nervous system can not achieve the effective blood drug concentration, and therefore are less active against disease in the central nervous system than against extra-cranial, systemic disease (8). Several chemotherapeutic agents in combination with WBRT have failed to result in the expected therapeutic benefit (9). Temozolomide (TMZ) is an oral, new alkylating agent that 
has demonstrated a preclinical activity against a variety of solid tumors. It readily crosses the BBB, achieving therapeutic concentrations in the brain, which makes it an attractive agent against BMs (10). It has been reported that the concomitant treatment of TMZ and WBRT was active and was conducive to improving quality of life (QoL) with an encouraging objective response (OR) rate (11). The combination of TMZ and WBRT may improve the treatment response (12).

We believe that adding TMZ to IMRT is more efficient than WBRT alone. The primary target of the study was to evaluate the recent response to treatment and safety, and the impact on QoL by using this treatment regimen with BMs from NSCLC.

\section{Patients and methods}

Eligibility criteria. Patients with histologically confirmed lung adenocarcinoma with no more than $4(\geq 1$ and $\leq 4)$ BMs by magnetic resonance imaging (MRI) and controlled extracranial disease between 2014 and 2015 were recruited. Patients were aged $\leq 75$ years, and had a World Health Organization Performance Status (PS) of $\leq 3$. Eligible patients may have received previous radiation therapy to the primary tumor or systemic metastatic sites but no previous WBRT or radiosurgery for BMs. Routine blood counts and biochemistry examination were basically normal. This study was approved by the Ethics Committee of the Affiliated Hospital of Soochow University (Suzhou, China). Signed informed consents were obtained from the patients.

Treatment plan. Every patient was immobilized by using a Medtec mask. A volumetric computed tomography simulation with a $3 \mathrm{~mm}$ slice thickness was required for planning of the target volume. Simulation computed tomography and pre-RT brain MRI fusion was performed for target delineation. The gross tumor volume (GTV) for BMs was delineated on the contrast-enhanced planning CT scan with visual comparison to the $\mathrm{T} 1+\mathrm{C}$-weighed axial diagnostic gadolinium-enhanced MRI (Gd-MRI) scan. Clinical tumor volume (CTV) was defined as the whole brain, and a margin of $5 \mathrm{~mm}$ was added to CTV as the planning target volume (PTV). The plan was created to deliver a dose of $30 \mathrm{~Gy}$ in 10 daily fractions to CTV according to IMRT, then additional dose of 9 Gy in 3 fractions of IMRT was delivered to GTV only. TMZ was administered orally, daily during RT at a dose of $75 \mathrm{mg} / \mathrm{m}^{2} /$ day from the first day to the end of day 14 . If grade $3 / 4$ toxicity occurred in the study, TMZ was withheld until the toxicity resolved, and the missed doses were no longer administered. For the control group, patients were given a dose of $30 \mathrm{~Gy}$ in 10 daily fractions to CTV according to IMRT, then additional dose of $9 \mathrm{~Gy}$ in 3 fractions of IMRT was delivered to GTV only without oral administration of temozolidomide.

Treatment evaluation and follow-up. Physical examination, neurological examination, QoL score and enhancement of the brain MRI were included in the pre-treatment evaluation within 28 days prior to treatment. Standard laboratory tests were obtained before treatment. All patients were monitored weekly during RT, including functional status assessment, neurologic examination, blood counts, biochemical functions and QoL score. Radiographic tumor response was assessed by Gd-MRI. Imaging was performed 3, 6 and 12 months following RT completion.

Patients were evaluated in the third month after treatment, including neurologic examination, functional status, toxicity assessment, QoL score and Gd-MRI of the brain. The primary endpoint was efficacy as measured by OR, including complete response (CR) and partial response (PR). CR was defined as a complete disappearance of all evidence of disease in the brain. PR was defined as response $>50 \%$ in all BMs. Responses in tumor lesions $<50 \%$ or increase in size $<25 \%$ was defined as stable disease (SD). A progressive disease (PD) was defined as either the occurrence of new lesion(s) or an increase in size of $>25 \%$. Tumor response for patients who died before the 3-month follow-up was defined as PD. PFS and overall survival (OS) were monitored.

Definition of progression and PFS. PFS was defined as the time from the date of the first dose of TMZ to the date of progression. Tumor progression was defined as an increment of $25 \%$ of the cross-product area measurement of nodular contrast enhancement compared with previous examination or any new enhancing tumor. Alternately, a clinical neurological performance change of two when causes other than tumor progression have been ruled out, was considered as progression.

Definition of OS. OS was defined as the time from the date of first dose of TMZ to date of death. Cause of death and its relationship with either the systemic or CNS disease was also recorded. Toxicity was evaluated according to the Common Terminology Criteria for Adverse Events (CTCAE) v3.0, divided into 1-5 degrees.

Evaluation of QoL. QoL was measured by using subject-completed Functional Assessment of Cancer Therapy (FACT) instrument. Twelve most relevant questions were selected in FACT questionnaire. The QoL was assessed around 3 months after radiotherapy. FACT for QoL score of cancer patients (1990 edition) includes appetite, spirit, sleep, fatigue, pain, family understanding and coordination, understanding and coordination of colleagues, understanding of cancer, attitude to treatment, daily life, side-effects of treatment, facial expression. It is divided into 5 levels, 1-5 the degree of expression is gradually reduced. QoL classification: the QoL full score is 60 points, poor QoL is $<20$ points, the difference is $21-30$ points, general is $31-40$ points, better is $41-50$ points, and good is $51-60$ points (13).

Statistical analysis. The analysis of treatment response and toxicity were evaluated in a descriptive manner. The descriptive method used in this report is to evaluate the short-term efficacy: according to the criteria for evaluating the curative effect of solid tumor, RECIST was used to evaluate the short-term curative effect, which was divided into $\mathrm{CR}, \mathrm{PR}, \mathrm{SD}$ and PD. CR: all the target lesions disappeared; PR: $30 \%$ reduction of the sum of the longest diameter of the target lesion compared with the baseline state; PD: the sum of the longest diameter of the target lesion increased by $20 \%$, or one or more new lesions, compared with the sum of the longest diameter of the target lesion recorded after the beginning of treatment; SD: between PR and PD. Objective response rate (ORR) includes CR and PR. The 
Table I. Patient characteristics and efficacy data evaluation after therapy.

\begin{tabular}{|c|c|c|}
\hline \multirow[b]{2}{*}{ Characteristics } & \multicolumn{2}{|c|}{ No. of patients $(\%)$} \\
\hline & $\mathrm{R}+\mathrm{T}$ & $\mathrm{R}$ \\
\hline \multicolumn{3}{|l|}{ Sex } \\
\hline Male & $7(77.8)$ & $6(66.7)$ \\
\hline Female & $2(22.2)$ & $3(33.3)$ \\
\hline \multicolumn{3}{|l|}{ Age (years) } \\
\hline$<40$ & $0(0)$ & $0(0)$ \\
\hline $40-60$ & $3(33.3)$ & $2(22.2)$ \\
\hline$>60$ & $6(66.7)$ & $7(77.8)$ \\
\hline \multicolumn{3}{|l|}{ ECOG score } \\
\hline 0 & $5(55.6)$ & $4(44.4)$ \\
\hline 1 & $4(44.4)$ & $5(55.6)$ \\
\hline \multicolumn{3}{|l|}{ No. of metastases } \\
\hline 1 & $3(33.3)$ & $3(33.3)$ \\
\hline 2 & $2(22.2)$ & $2(22.2)$ \\
\hline 3 & $0(0)$ & $2(22.2)$ \\
\hline 4 & $4(44.4)$ & $2(22.2)$ \\
\hline \multicolumn{3}{|l|}{ Year of recruitment } \\
\hline 2014 & $3(33.3)$ & $1(11.1)$ \\
\hline 2015 & $6(66.7)$ & $8(88.9)$ \\
\hline \multicolumn{3}{|l|}{ Time course of disease (months) } \\
\hline$<1$ & $0(0)$ & $0(0)$ \\
\hline $1-3$ & $0(0)$ & $1(11.1)$ \\
\hline $3-6$ & $1(11.1)$ & $2(22.2)$ \\
\hline $6-12$ & $4(44.4)$ & $3(33.3)$ \\
\hline$\geq 12$ & $4(44.4)$ & $3(33.3)$ \\
\hline \multicolumn{3}{|l|}{ Response } \\
\hline $\mathrm{CR}$ & $1(11.1)$ & $0(0)$ \\
\hline PR & $6(66.7)$ & $4(44.4)$ \\
\hline Objective response $(\mathrm{CR}+\mathrm{PR})$ & $7(77.8)$ & $4(44.4)$ \\
\hline $\mathrm{SD}$ & $2(22.2)$ & $3(33.3)$ \\
\hline Local control (CR+PR+SD) & $9(100)$ & $7(77.8)$ \\
\hline $\mathrm{PD}$ & $0(0)$ & $2(22.2)$ \\
\hline
\end{tabular}

$\mathrm{CR}$, complete response; $\mathrm{PR}$, partial response; $\mathrm{SD}$, stable disease; $\mathrm{PD}$, progressive disease.

descriptive method used in adverse reactions was classified into 0-4 grades according to the commonly used drug toxicity standard CTCAE v3.0. Kaplan-Meier estimates were used to assess PFS and OS. Log-rank test was used for survival analysis. The QoL data were analysed by ANOVA test and the post hoc test was SNK. SPSS software (version 19.00; IBM Corp., Armonk, NY, USA) was used for statistical analysis.

\section{Results}

Between 2014 and 2016, 18 patients were enrolled. The demographics and baseline disease characteristics of the assessable patients are listed in Table I.
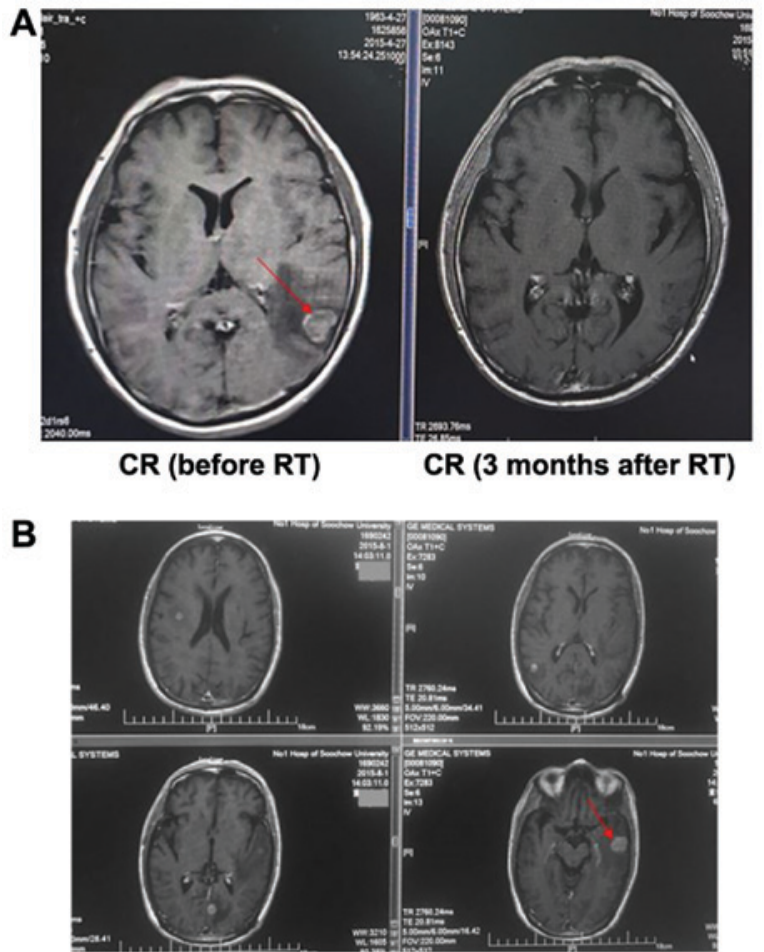

PR (before RT)

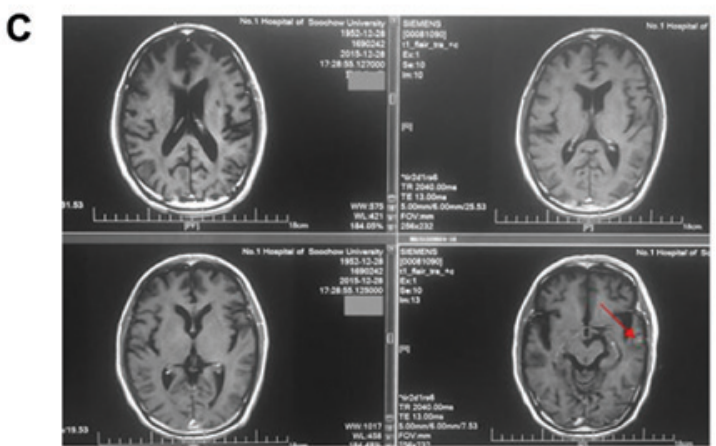

PR (3 months after RT)

Figure 1. The arrow indicates the BM of lung cancer; 3 months after RT, PR occurred and the tumor shrank. (A) CR before RT and 3 months after RT; (B) PR before RT; (C) PR 3 months after RT. BM, brain metastasis; PR, partial response; $\mathrm{CR}$, complete response.

Response of BMs after therapy. Objective tumor response was evaluated after 3 months. For the combined treatment group, 1 patient achieved CR (11.1\%), 6 patients obtained PR $(66.7 \%)$, and there were no patients in progression. Therefore, as shown in Table I, OR reached $77.8 \%$. While the disease local control $(\mathrm{CR}+\mathrm{PR}+\mathrm{SD})$ rate obtained $100 \%$. However, the other 9 patients who received intensity-modulated radiation only had much worse results. The $\mathrm{CR}$ was $0, \mathrm{PR}$ rate was $44.4 \%$, OR rate was $44.4 \%$ (Table I). The MRI of a patient is shown in Fig. 1.

Safety and tolerability. Acute adverse effects are shown in Table II. Compared to control group, adding TMZ to IMRT was generally well tolerated. The most common side-effect was anemia. The most frequent adverse events include neutropenia, anemia, nausea, fatigue, vomiting, anorexia and dizziness. Most side-effects can be alleviated and controlled by supporting therapy. 
Table II. Adverse events during treatment.

No. of No. of

Toxicity Grade 1-2 Grade 3 Grade $\geq 4$ patients $(\mathrm{R}+\mathrm{T}) \quad$ Grade $1-2 \quad$ Grade $3 \quad$ Grade $\geq 4 \quad$ patients $(\mathrm{R})$

\begin{tabular}{|c|c|c|c|c|c|c|c|c|}
\hline Neutropenia & 3 & 0 & 0 & $3(33.3 \%)$ & 2 & 0 & 0 & $2(22.2 \%)$ \\
\hline Thrombocytopenia & 2 & 0 & 0 & $2(22.2 \%)$ & 2 & 0 & 0 & $2(22.2 \%)$ \\
\hline Anemia & 4 & 0 & 0 & $4(44.4 \%)$ & 3 & 0 & 0 & $3(33.3 \%)$ \\
\hline Nausea & 1 & 1 & 0 & $2(22.2 \%)$ & 1 & 1 & 0 & $2(22.2 \%)$ \\
\hline Vomiting & 2 & 1 & 0 & $3(33.3 \%)$ & 2 & 0 & 0 & $2(22.2 \%)$ \\
\hline Fatigue & 3 & 0 & 0 & $3(33.3 \%)$ & 2 & 0 & 0 & $2(22.2 \%)$ \\
\hline Anorexia & 2 & 0 & 0 & $2(22.2 \%)$ & 2 & 0 & 0 & $2(22.2 \%)$ \\
\hline Dizziness & 2 & 0 & 0 & $2(22.2 \%)$ & 1 & 0 & 0 & $1(11.1 \%)$ \\
\hline
\end{tabular}

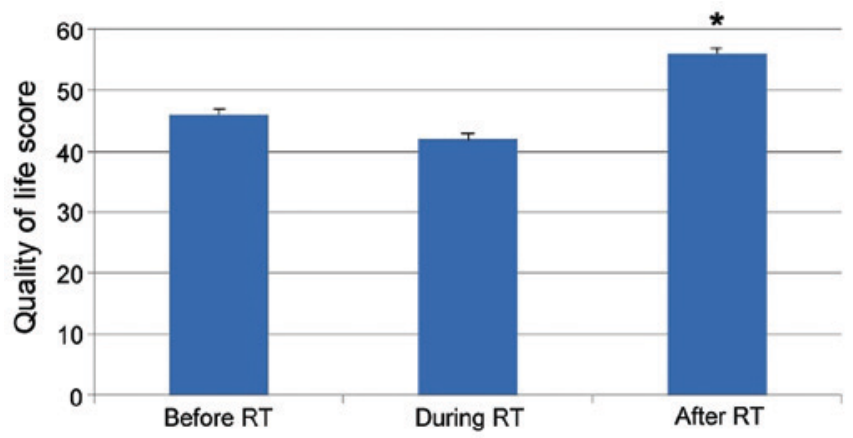

Figure 2. The QoL before, during and after RT. QoL is the QoL score which was assessed in the third month after treatment. Higher QoL means better life quality. Before and during RT, the patients received special care and were not able to take care of themselves. Their QoL scores were just $>40$. After RT, the self-care ability of patients was improved, and the QoL increased to $\sim 56$. ${ }^{*} \mathrm{P}<0.05$, compared with before and during RT. QoL, quality of life.

Impact on QoL. Eighteen patients completed the assessment of QoL questionnaire. The mean QoL-score after 3 months was significantly improved, and there were significant differences $(\mathrm{p} \leq 0.05)$. The change of the mean QoL-score is shown before treatment, during treatment, after treatment in Fig. 2.

Survival analysis. Compared with the control group, the combined treatment group had better OS rate and no progression-free survival time, however, the difference was not statistically significant due to the small number of cases $(\mathrm{p}=0.390,0.281)($ Fig. 3).

\section{Discussion}

BMs are a major cause of mortality in patients with NSCLC. In general, the results of a previous analysis had confirmed the advantages of IMRT. On the one hand, IMRT boosted greater biological anticancer effectiveness, due to the accelerated treatment at GTV. On the other hand, it also improved sparing of healthy brain tissue, due to the integration of the boost within the WBRT treatment. A case of a patient with 8 BMs was published (14). By IMRT and IMRT-simultaneous integrated boost (SIB), maximum doses were $<105 \%$ of prescribed dose, $95 \%$ prescribed dose contained the volume of for all BMs and dose homogeneity is within 3\%. Moreover,
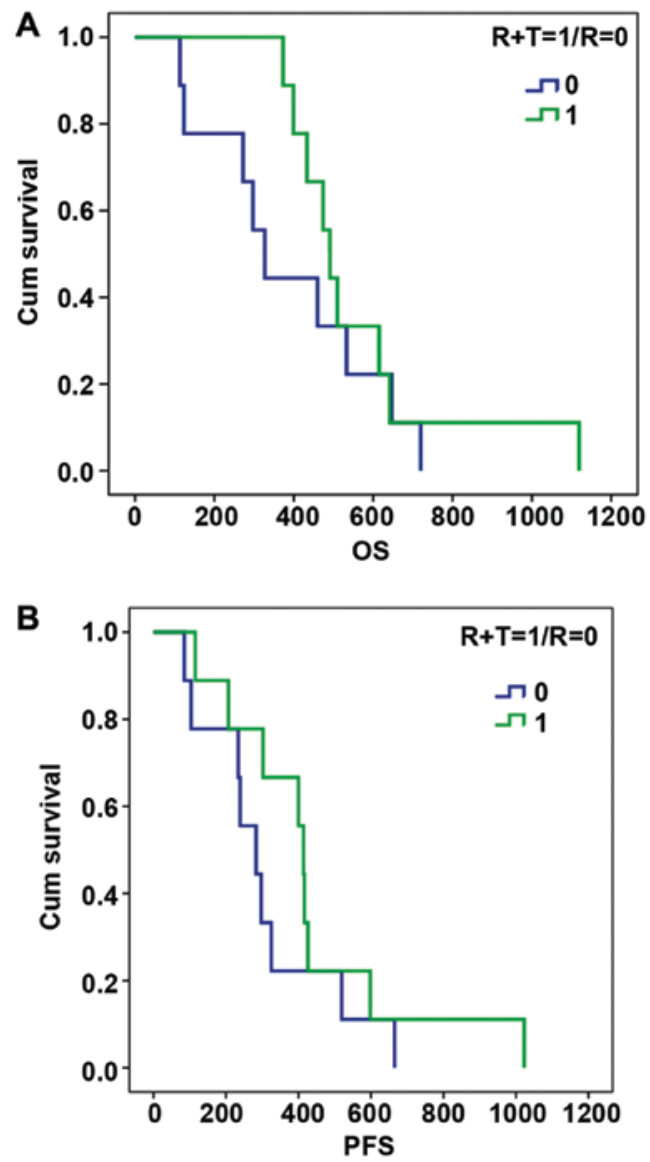

Figure 3. Survival analysis. (A) A total of 1,200 days of OS rate for control and combination treatment group; (B) 1,200 days of PFS rate for control and combination treatment group. OS, overall survival.

maximum doses to eyes, lens, optic nerves and optic chiasma were limited. It contributed to the protection of normal organs. From a clinical point of view, subsequent MRI brain controls showed a complete clinical response. After 40 months of treatment, the patients had no PD and showed no late brain toxicity. A decision was reached that the use of concomitant boost treatment by IMRT during routine WBRT improved overall local control and had no adverse effects without resort to Gamma Knife SRS $(15)$. Some studies $(16,17)$ thought that IMRT could conformally avoid the hippocampus, which is an 
important part of the brain memory function. In the study we report 18 patients with a limited number $(\leq 4)$ of BMs in whom an IMRT facility has been used to sequentially boost the GTV of bulky brain metastatic disease to $(39 \mathrm{~Gy} / 13 \mathrm{~F} / 3 \mathrm{~W})$ after a (30 Gy/10 F/2 W). The volume for all lesions receiving $95 \%$ of prescribed dose was boosted. IMRT could guarantee a better $\mathrm{CI}$ and HI. Moreover, IMRT plan can significantly reduce the irradiation dose of eyes, lens, optic, nerves, and parotid glands, and especially the middle ear.

Chemotherapy undoubtedly remains the primary therapeutic approach for disseminated systemic tumor, and therefore it is likely to be a reasonable choice for BMs of NSCLC as well. Radiotherapy combined with chemotherapy turned to primary research tendency in recent years. Some clinical trials (18-22) failed to explore effective drug to improve OR rate and prolong survival for BMs of NSCLC, for example carboplatin, cisplatin, 5-FU, topotecan, and vinorelbine. One cause involved the ability of chemotherapeutic agents to cross the BBB, therefore limited the delivery of chemotherapeutic agents into the brain and could not achieve therapeutic blood concentration. However, several chemotherapeutic agents has been developed with the ability to cross BBB, but only a few reached the clinical phase of development. TMZ was the best used and studied in clinic, because TMZ easily penetrates $\mathrm{BBB}$, and oral, secure, and low toxic profile. TMZ is an alkylating agent prodrug, delivering a methyl group to purine bases of DNA (O6-guanine; N7-guanine and N3-adenine). During DNA replication, the methylated guanines or adenines would mispair with thymine or cytosine, which would induce DNA damage and toxic lesion, then cell apoptosis (23). It was reported that (24) 32 cases of NSCLC with BM were treated with WBRT followed by IMBRT with concomitant TMZ. The results showed that the OR rate was $37.5 \%$, the median survival time was 8 months, and the median PFS was 5.5 months. Toxic reaction was well tolerated.

In 2007, study showed that (11) 59 patients were enrolled and received 30 Gy WBRT with concomitant TMZ (75 mg/m²/day) for 10 days, and subsequently continued to take TMZ (150 mg/m²/day) for six cycles. Five patients achieved a CR, 21 patients obtained a PR, while 18 patients had SD. The overall response rate $(45 \%)$ exceeded the target activity per study design. The median OS was 13 months, and the median time to progression was 9 months. The treatment is well tolerated, with an remarkable OR rate, and a significant improvement in QoL $(\mathrm{p}<0.0001)$. A single-institution phase II clinical trial was reported (25). Twenty-seven cases were treated with WBRT (30 Gy/10 F/2 W) with concomitant TMZ ( $75 \mathrm{mg} / \mathrm{m}^{2} /$ day) for 10 days, and then continued to take low dose TMZ (75 mg/m²/day) for 21 days/month, for up to 12 cycles. Two patients obtained CR (7.4\%), and 11 patients had a PR (40.7\%). The overall median survival time and median progression-free time, respectively, were 8.8 and 6 months, and the treatment was well tolerated. It could be concluded that WBRT combined with long-term low dose TMZ appeared to be an effective, well-tolerated regimen.

A randomised phase II study evaluated the use of TMZ concomitant with WBRT (30 Gy/10 F/2 W) and WBRT alone in patients with BMs (26). The OR of the TMZ plus WBRT was $78.6 \%$, and only $48.1 \%$ for WBRT alone. The median PFS in combination group and WBRT alone was 11.8 and 5.6 months, respectively. However, there was no significant difference in OS between the two groups.

Moreover, two randomized phase II studies compared TMZ given concurrently with WBRT and WBRT alone in patients with BMs. Both studies showed an improved overall response rate in the combination arm, resulting in a benefit in progression-free survival in only one study $(27,28)$. However, some reports $(29,30)$ also indicate that TMZ and concurrent treatment with radiation did not produce better outcomes, including response rates and longer-term tumor control. Besides, from the very recent study results, memantine, MAP-kinase inhibitors and Rac inhibitors also showed very positive data in the trials, which remind us to replace TMZ with these drugs for further combination treatment study $(16,31,32)$. Due to the difference in the time and position during examination, it is unlikely to provide equivalent slice levels to allow comparison of pre- and post-therapy response, we will try to make improvement in the following study.

Overall evidence and future directions: the reported trial demonstrated that concomitant treatment with IMRT and TMZ (at a dose of $75 \mathrm{mg} / \mathrm{m}^{2} /$ day for 14 days) was well tolerated and active, achieving a CR rate of $29 \%$ and an objective rate of $100 \%$. We demonstrated a positive impact of this therapy on the QoL of patients with BMs after 3 months from the initiation of treatment with TMZ $(\mathrm{p}<0.05)$. However, this study has limitations because of the sample size, and further case study will be needed.

\section{Acknowledgements}

Not applicable.

\section{Funding}

No funding was received.

\section{Availability of data and materials}

The datasets used and/or analyzed during the present study are available from the corresponding author on reasonable request.

\section{Authors' contributions}

JL, JW and YW conceived and designed the study. JL, XC, YC, $\mathrm{XH}$ and $\mathrm{HZ}$ were responsible for the collection and analysis of the patient data. JL, XC, JW and YW interpreted the data and drafted the manuscript. YW revised the manuscript critically for important intellectual content. All authors have read and approved the final manuscript.

\section{Ethics approval and consent to participate}

The study was approved by the Ethics Committee of the Affiliated Hospital of Soochow University (Suzhou, China). Signed informed consents were obtained from the patients or the guardians.

\section{Patient consent for publication}

Not applicable. 


\section{Competing interests}

The authors declare that they have no competing interests.

\section{References}

1. Carbone DP, Ding K, Roder H, Grigorieva J, Roder J, Tsao MS, Seymour L and Shepherd FA: Prognostic and predictive role of the VeriStrat plasma test in patients with advanced non-small-cell lung cancer treated with erlotinib or placebo in the NCIC Clinical Trials Group BR.21 trial. J Thorac Oncol 7: 1653-1660, 2012.

2. Gaspar LE, Mehta MP, Patchell RA, Burri SH, Robinson PD, Morris RE, Ammirati M, Andrews DW, Asher AL, Cobbs CS, et al: The role of whole brain radiation therapy in the management of newly diagnosed brain metastases: A systematic review and evidence-based clinical practice guideline. J Neurooncol 96 : 17-32, 2010.

3. Bhatnagar AK, Flickinger JC, Kondziolka D and Lunsford LD: Stereotactic radiosurgery for four or more intracranial metastases. Int J Radiat Oncol Biol Phys 64: 898-903, 2006.

4. Kirby N, Chuang C, Pouliot J, Hwang A and Barani IJ: Physics strategies for sparing neural stem cells during whole-brain radiation treatments. Med Phys 38: 5338-5344, 2011.

5. Tarnawski R, Michalecki L, Blamek S, Hawrylewicz L, Piotrowski T, Slosarek K, Kulik R and Bobek-Billewicz B: Feasibility of reducing the irradiation dose in regions of active neurogenesis for prophylactic cranial irradiation in patients with small-cell lung cancer. Neoplasma 58: 507-515, 2011.

6. Gondi V, Tolakanahalli R, Mehta MP, Tewatia D, Rowley H, Kuo JS, Khuntia D and Tomé WA: Hippocampal-sparing whole-brain radiotherapy: A 'how-to' technique using helical tomotherapy and linear accelerator-based intensity-modulated radiotherapy. Int J Radiat Oncol Biol Phys 78: 1244-1252, 2010.

7. Wolff D, Abo-Madyan Y, Dobler B, Lohr F, Mai S, Polednik M and Wenz F: Serial tomotherapy vs. MLC-IMRT (multileaf collimator intensity modulated radiotherapy) for simultaneous boost treatment large intracerebral lesions. Z Med Phys 19: 58-66, 2009 (In German).

8. Langer CJ and Mehta MP: Current management of brain metastases, with a focus on systemic options. J Clin Oncol 23: 6207-6219, 2005.

9. Mehta MP, Paleologos NA, Mikkelsen T, Robinson PD, Ammirati M, Andrews DW, Asher AL, Burri SH, Cobbs CS, Gaspar LE, et al: The role of chemotherapy in the management of newly diagnosed brain metastases: A systematic review and evidence-based clinical practice guideline. J Neurooncol 96: 71-83, 2010.

10. Tatar Z, Thivat E, Planchat E, Gimbergues P, Gadea E, Abrial C and Durando X: Temozolomide and unusual indications: Review of literature. Cancer Treat Rev 39: 125-135, 2013.

11. Addeo R, Caraglia M, Faiola V, Capasso E, Vincenzi B, Montella L, Guarrasi R, Caserta L and Del Prete S: Concomitant treatment of brain metastasis with whole brain radiotherapy [WBRT] and temozolomide [TMZ] is active and improves quality of life. BMC Cancer 7: 18, 2007.

12. Liu R, Wang X, Ma B, Yang K, Zhang Q and Tian J: Concomitant or adjuvant temozolomide with whole-brain irradiation for brain metastases: A meta-analysis. Anticancer Drugs 21: 120-128, 2010.

13. EuroQol Group: EuroQol - a new facility for the measurement of health-related quality of life. Health Policy 16: 199-208, 1990

14. Ferro M, Cilla S, Macchia G, Deodato F, Pierro A, Digesu C, Ferrandina G, Ciuffreda M, Sallustio G and Morganti AG: On the cutting edge of intensity modulated radiotherapy and simultaneous integrated boost (IMRT-SIB): The case of a patient with 8 brain metastases. Rep Pract Oncol Radiother 20: 316-319, 2015.

15. Edwards AA, Keggin E and Plowman PN: The developing role for intensity-modulated radiation therapy (IMRT) in the non-surgical treatment of brain metastases. Br J Radiol 83: 133-136, 2010.

16. Slade AN and Stanic S: The impact of RTOG 0614 and RTOG 0933 trials in routine clinical practice: The US Survey of Utilization of Memantine and IMRT planning for hippocampus sparing in patients receiving whole brain radiotherapy for brain metastases. Contemp Clin Trials 47: 74-77, 2016.
17. Gondi V, Pugh SL, Tome WA, Caine C, Corn B, Kanner A, Rowley H, Kundapur V, DeNittis A, Greenspoon JN, et al: Preservation of memory with conformal avoidance of the hippocampal neural stem-cell compartment during whole-brain radiotherapy for brain metastases (RTOG 0933): A phase II multi-institutional trial. J Clin Oncol 32: 3810-3816, 2014.

18. Guerrieri M, Wong K, Ryan G, Millward M, Quong G and Ball DL: A randomised phase III study of palliative radiation with concomitant carboplatin for brain metastases from non-small cell carcinoma of the lung. Lung Cancer 46: 107-111, 2004.

19. Robinet G, Thomas P, Breton JL, Léna H, Gouva S, Dabouis G, Bennouna J, Souquet PJ, Balmes P, Thiberville L, et al: Results of a phase III study of early versus delayed whole brain radiotherapy with concurrent cisplatin and vinorelbine combination in inoperable brain metastasis of non-small-cell lung cancer: Groupe Français de Pneumo-Cancérologie (GFPC) Protocol 95-1. Ann Oncol 12: 59-67, 2001.

20. Lee DH, Han JY, Kim HT, Yoon SJ, Pyo HR, Cho KH, Shin SH, Yoo H, Lee SH and Lee JS: Primary chemotherapy for newly diagnosed nonsmall cell lung cancer patients with synchronous brain metastases compared with whole-brain radiotherapy administered first: Result of a randomized pilot study. Cancer 113: 143-149, 2008.

21. Kim DY, Lee KW, Yun T, Kim DW, Kim TY, Heo DS, Bang YJ and Kim NK: Efficacy of platinum-based chemotherapy after cranial radiation in patients with brain metastasis from non-small cell lung cancer. Oncol Rep 14: 207-211, 2005.

22. Neuhaus T, Ko Y, Muller RP, Grabenbauer GG, Hedde JP, Schueller H, Kocher M, Stier S and Fietkau R: A phase III trial of topotecan and whole brain radiation therapy for patients with CNS-metastases due to lung cancer. Br J Cancer 100: 291-297, 2009.

23. Zhang J, Stevens MF and Bradshaw TD: Temozolomide: Mechanisms of action, repair and resistance. Curr Mol Pharmacol 5: 102-114, 2012 .

24. Wang Q, Jiang Z, Qi X, Lu S, Wang S, Leng C, Lu F, Liu H, Liang $S$ and Shi J: Whole brain radiation therapy followed by intensity-modulated boosting treatment combined with concomitant temozolomide for brain metastases from non-small-cell lung cancer. Clin Transl Oncol 16: 1000-1005, 2014.

25. Addeo R, De Rosa C, Faiola V, Leo L, Cennamo G, Montella L, Guarrasi R, Vincenzi B, Caraglia M and Del Prete S: Phase 2 trial of temozolomide using protracted low-dose and whole-brain radiotherapy for nonsmall cell lung cancer and breast cancer patients with brain metastases. Cancer 113: 2524-2531, 2008.

26. Gamboa-Vignolle C, Ferrari-Carballo T, Arrieta Ó and Mohar A: Whole-brain irradiation with concomitant daily fixed-dose temozolomide for brain metastases treatment: A randomised phase II trial. Radiother Oncol 102: 187-191, 2012

27. Verger E, Gil M, Yaya R, Viñolas N, Villà S, Pujol T, Quintó L and Graus F: Temozolomide and concomitant whole brain radiotherapy in patients with brain metastases: A phase II randomized trial. Int J Radiat Oncol Biol Phys 61: 185-191, 2005.

28. Antonadou D, Paraskevaidis M, Sarris G, Coliarakis N, Economou I, Karageorgis P and Throuvalas N: Phase II randomized trial of temozolomide and concurrent radiotherapy in patients with brain metastases. J Clin Oncol 20: 3644-3650, 2002.

29. Hassler MR, Pfeifer W, Knocke-Abulesz TH, Geissler K, Altorjai G, Dieckmann K and Marosi C: Temozolomide added to whole brain radiotherapy in patients with multiple brain metastases of non-small-cell lung cancer: A multicentric Austrian phase II study. Wien Klin Wochenschr 125: 481-486, 2013.

30. Chua D, Krzakowski M, Chouaid C, Pallotta MG, Martinez JI, Gottfried M, Curran W and Throuvalas N: Whole-brain radiation therapy plus concomitant temozolomide for the treatment of brain metastases from non-small-cell lung cancer: A randomized, open-label phase II study. Clin Lung Cancer 11: 176-181, 2010.

31. Hamilton $\mathrm{E}$ and Infante JR: Targeting CDK4/6 in patients with cancer. Cancer Treat Rev 45: 129-138, 2016.

32. Cardama GA, Gonzalez N, Maggio J, Menna PL and Gomez DE: Rho GTPases as therapeutic targets in cancer (Review). Int J Oncol 51: 1025-1034, 2017.

This work is licensed under a Creative Commons Attribution-NonCommercial-NoDerivatives 4.0 International (CC BY-NC-ND 4.0) License. 